\title{
Ising model with stochastic resetting
}

\author{
Matteo Magoni $\odot,{ }^{1,2}$ Satya N. Majumdar, ${ }^{1}$ and Grégory Schehr ${ }^{1}$ \\ ${ }^{1}$ Université Paris-Sud, CNRS, LPTMS, UMR 8626, 91405 Orsay, France \\ ${ }^{2}$ Department of Applied Science and Technology, Politecnico di Torino, Corso Duca degli Abruzzi 24, 10129 Torino, Italy
}

(Received 13 February 2020; accepted 28 May 2020; published 3 August 2020)

\begin{abstract}
We study the nonequilibrium stationary state (NESS) of the Ising model driven away from thermal equilibrium at temperature $T$ by a stochastic resetting protocol. This protocol, realizable via rapid quench of temperature and magnetic field, resets the magnetization to its fixed initial value $m_{0}$ at a constant rate $r$. In the resulting NESS, the magnetization acquires a nontrivial distribution, leading to a rich phase diagram in the $(T, r)$ plane. We establish these results exactly in one dimension and present scaling arguments supported by simulations in two dimensions. We show that this resetting protocol gives rise to a "pseudoferro" phase in the $(T, r)$ plane for $r>r^{*}(T)$ and $T>T_{c}$, where $r^{*}(T)$ is a crossover line separating the pseudoferro phase from a paramagnetic phase. This pseudoferro phase is characterized by a magnetization distribution that vanishes as $\sim m^{\zeta}$ as $m \rightarrow 0$, where $\zeta$ varies continuously with $T$ and $r$.
\end{abstract}

DOI: 10.1103/PhysRevResearch.2.033182

\section{INTRODUCTION AND MAIN RESULTS}

Stochastic resetting has seen much activity during the past decade [1], notably in the context of search processes which are ubiquitous in nature [2]. The simple intuition behind resetting is as follows: If one is searching for a target via a stochastic process such as simple diffusion, it may take a long time due to trajectories that run off from the target. It is then advantageous to restart the search process with a certain resetting rate $r$ from the same initial condition $[3,4]$. The idea is that one may explore new pathways leading to the target, thereby reducing the search time. Recently, a large number of studies have shown that there is an optimal resetting rate $r^{*}$ that makes the search process most efficient [3-21]. Another interesting aspect of resetting dynamics, in addition to optimizing the search process, is that it breaks detailed balance and drives the system into a nontrivial nonequilibrium stationary state (NESS). Characterizing such a NESS has recently become a problem of central interest in statistical physics [3,22-27].

Resetting has found a large number of applications across disciplines. For example, in biology, the process of RNA polymerization, which is responsible for the synthesis of RNA from a DNA template, is stochastically interrupted by backtracking [28,29]. Similar notions are found in the ecological context: Animals such as rhesus monkeys [30,31], during the foraging period, perform stochastic resetting to previously visited sites and the effects of such memory-induced resetting have been studied in several models [18,24,32-35]. In computer science, stochastic restarts can be used to reduce

Published by the American Physical Society under the terms of the Creative Commons Attribution 4.0 International license. Further distribution of this work must maintain attribution to the author(s) and the published article's title, journal citation, and DOI. the running time of randomized search algorithms [36-41]. Stochastic resetting has been studied also in the context of chemical processes as in the Michaelis-Menton reaction scheme [9,12], active run-and-tumble particles [42-44], biological traffic models [45], population genetics [46], and recently in quantum systems $[47,48]$.

Most of the systems discussed above concern the effect of resetting on the statics and dynamics of a single particle (or equivalently for noninteracting systems). It is natural to ask how resetting affects the stationary state of a many-body interacting system. This question has been addressed in a number of interacting systems in one dimension, such as reaction-diffusion systems [49], fluctuating interfaces [50], exclusion and zero-range processes subjected to resetting [51-53]. However, none of these systems, in equilibrium, exhibit a thermodynamic phase transition. It is then interesting to know how resetting affects a system that, in the absence of resetting, displays a phase transition in its equilibrium state. The simplest paradigmatic model that exhibits an equilibrium phase transition is the Ising model in $d$ dimensions, with $d \geqslant 2$. It is then natural to ask what kind of stationary state is reached when an Ising model, evolving under the natural Glauber dynamics, is subject to resetting (to an initial state) at a constant rate $r$ ?

It is useful to first recall the properties of the nearest neighbor Glauber-Ising model in the absence of resetting ( $r=0$ ) [54]. Let $s_{i}= \pm 1$ denote the spin at site $i$ of the Ising model. Starting from an initial configuration where the spins are independently chosen to have value \pm 1 with probability $\left(1 \pm m_{0}\right) / 2$ (where $\left.m_{0} \in[0,1]\right)$, the individual spins flip according to the Glauber rate that satisfies detailed balance (see later for details). Let $P\left(\left\{s_{i}\right\}, t\right)$ denote the probability distribution of a spin configuration $\left\{s_{i}\right\}$ at time $t$. The most natural observable is the order parameter, i.e., the average magnetization $m(t)=\frac{1}{N} \sum_{i}\left\langle s_{i}(t)\right\rangle$, where $\langle\cdots\rangle$ denotes averaging over the probability measure $P\left(\left\{s_{i}\right\}, t\right)$ at time $t$. At late times, the system approaches the thermal equilibrium 
state, where the magnetization $m(t)$ approaches, irrespective of the initial value $m_{0}$, the final value $m_{\mathrm{eq}}>0$ for $T<T_{c}$ (ferromagnetic phase), and 0 for $T \geqslant T_{c}$ (paramagnetic phase and at the critical point). Of course, $T_{c}=0$ in $d=1$.

What happens to the magnetization $m(t)$ at long times when a finite resetting rate $r$ is switched on? Resetting can be physically realized by the following "rapid quench" protocol. We prepare the Ising model initially in its equilibrium state at high temperature (say $T=\infty$ ) in the presence of a nonzero magnetic field $h>0$. So, at $t=0$, the spins are uncorrelated with a finite magnetization $m_{0}$. We rapidly quench the temperature to $T$ and switch off the magnetic field and let the system evolve via the Glauber dynamics at temperature $T$ and $h=0$ during a random time distributed exponentially $p(t)=r e^{-r t}$. Following this period, we do a reverse quench by raising the temperature to $T=\infty$ and switching on $h>0$. We let the system relax to its equilibrium over a relaxation time $\tau_{R}$, and then again quench the system back to $T$ and $h=0$, run it for an exponential time and do reverse quench and so on. This alternating quench-and-reverse-quench protocol mimics exactly the resetting mechanism in the presence of a finite refraction time $\tau_{R}$ that has been studied recently in various models of stochastic resetting [15,55-57]. At very high temperature, we expect rapid relaxation $\tau_{R} \ll 1 / r$. This approximation amounts to instantaneous resetting $\tau_{R} \simeq 0$, to which we focus here as it is easier to analyze.

In this paper, we show that this resetting protocol drives the system into a NESS where the magnetization has a nontrivial distribution. This is markedly different from equilibrium systems where the magnetization distribution is trivially a $\delta$ function centred either at $m=0$ (for $T>T_{c}$ ) or at $m=m_{\mathrm{eq}}>$ 0 (for $T<T_{c}$ ). Thus, the knowledge of the full distribution of the magnetization is necessary to characterize the steady state of the system in the presence of resetting.

Let us first summarize our main results. We show that a nonzero resetting leads to a rich phase diagram in the $(T, r)$ plane as displayed in Fig. 1 with the emergence of a "pseudoferro" phase. At all $T$, the stationary magnetization distribution $P_{r}^{\text {stat }}(m)$ has a finite support. For $T>T_{c}$, there is a crossover line $r^{*}(T)$ that separates the paramagnetic phase $\left[r<r^{*}(T)\right]$ from a pseudoferro phase $\left[r>r^{*}(T)\right]$. In the para phase, $P_{r}^{\text {stat }}(m)$ diverges as $m \rightarrow 0$, as $P_{r}^{\text {stat }}(m) \sim m^{\zeta}$ with $\zeta=r / r^{*}(T)-1<0$ and hence the typical magnetization $m_{\text {typ }}=0\left(m_{\text {typ }}\right.$ denotes the value of $m$ at which $P_{r}^{\text {stat }}(m)$ has its maximum). In addition, there is no gap at $m=0$, i.e., $g=0$. In contrast, in the pseudoferro phase $\left[T>T_{c}\right.$ and $\left.r>r^{*}(T)\right]$, while the gap $g$ still remains zero, $P_{r}^{\text {stat }}(m)$ now vanishes as $m \rightarrow 0$ as $P_{r}^{\text {stat }}(m) \sim m^{\zeta}$ where the exponent $\zeta=r / r^{*}(T)-$ $1>0$ varies continuously with $T$ and $r$. Consequently, the maximum of $P_{r}^{\text {stat }}(m)$ occurs at a nonzero value $m_{\text {typ }}>0$ (see Fig. 1). For $T<T_{c}$ (ferro phase), the distribution $P_{r}^{\text {stat }}(m)$ has a finite support $\left[m_{\mathrm{eq}}, m_{0}\right]$ (for $m_{\mathrm{eq}}<m_{0}$ ) or over $\left[m_{0}, m_{\mathrm{eq}}\right]$ (if $m_{\mathrm{eq}}>m_{0}$ ). Thus, in this phase, there is a finite nonzero gap $g=\min \left(m_{\mathrm{eq}}, m_{0}\right)$. In addition, $m_{\mathrm{typ}}>0$ in the ferro phase. Exactly at $T=T_{c}$, the distribution $P_{r}^{\text {stat }}(m)$ vanishes extremely rapidly, $P_{r}^{\text {stat }}(m) \sim e^{-A m^{-\kappa}}$ as $m \rightarrow 0$. We show that the exponent $\kappa$ is related to the equilibrium critical exponents via the relation $\kappa=v z / \beta$, where $\nu$ and $\beta$ are respectively the correlation length and the order parameter critical exponents,

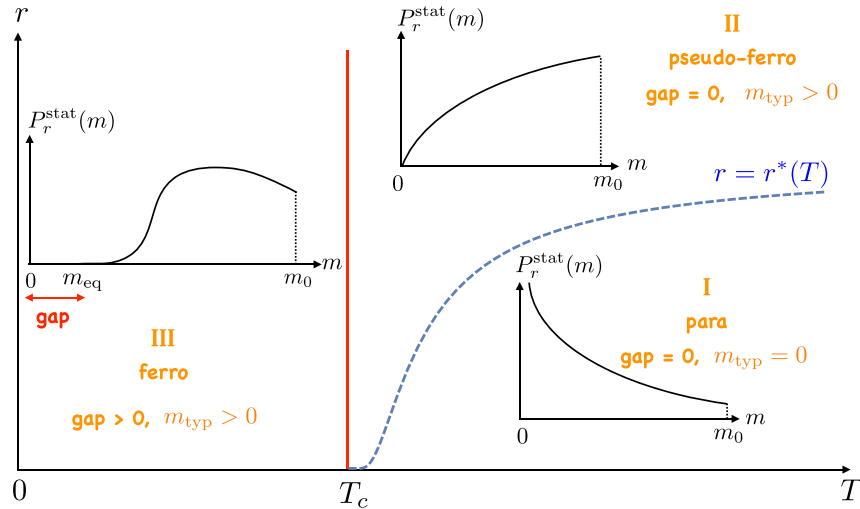

FIG. 1 . Phase diagram in the $(T, r)$ plane in $d \geqslant 2$. The magnetization distribution $P_{r}^{\text {stat }}(m)$ in the stationary state, in the presence of resetting, is shown schematically in three different regions in the $(T, r)$ plane. For $T>T_{c}$, there is a crossover line $r^{*}(T)$ (shown schematically by the dashed blue line) that separates the para phase for $r<r^{*}(T)$ and the pseudoferro phase for $r>r^{*}(T)$. In the para phase, the $P_{r}^{\text {stat }}(m)$ has a divergent peak at $m=m_{\text {typ }}=0$ (and no gap at $m=0)$, while in the pseudoferro phase, $P_{r}^{\text {stat }}(m)$ vanishes at $m=0$ with a peak at $m_{\text {typ }}>0$ (and still no gap at $m=0$ ). For $T<T_{c}$ (ferro phase), a nonzero gap opens up at $m=0$ in $P_{r}^{\text {stat }}(m)$ and moreover the distribution peaks at $m_{\mathrm{typ}}>0$.

while $z$ is the dynamical critical exponent associated to the Ising-Glauber dynamics at $T=T_{c}$. We establish these results from an exact solution in $d=1$ and, for $d=2$, we provide scaling arguments supported by simulations.

\section{RESETTING IN THE ISING MODEL}

We start with an Ising model with ferromagnetic nearestneighbor interactions $H=-J \sum_{\langle i, j\rangle} s_{i} s_{j}$ on a $d$-dimensional lattice with $N$ sites and periodic boundary conditions. Starting from an initial condition where the spins are independently \pm 1 with probability $\left(1 \pm m_{0}\right) / 2$, the Glauber dynamics, in the absence of resetting, consists in flipping a single spin with rate [54]

$$
w\left(s_{i} \rightarrow-s_{i}\right)=\frac{1}{1+e^{\beta \Delta E}},
$$

where $\beta=1 /\left(k_{B} T\right)$ is the inverse temperature and $\Delta E=$ $2 J s_{i} \sum_{j \in \text { n.n. }} s_{j}$ is the change of energy in flipping the $i$ th spin. In $d=1$, this rate simplifies to $w\left(s_{i} \rightarrow-s_{i}\right)=(1 / 2)[1-$ $\left.\gamma s_{i}\left(s_{i-1}+s_{i+1}\right) / 2\right]$, where $\gamma=\tanh (2 \beta J)$. This property makes the one-dimensional ( $1 d$ ) Glauber dynamics exactly solvable as the evolution equation for the $n$-point correlation functions only involve $n$-point functions; i.e., it satisfies a closure property [54]. This closure property, however, does not hold for $d>1$. Note that under the Glauber dynamics, the magnetization $m(t)=(1 / N) \sum_{i}\left\langle s_{i}(t)\right\rangle$ evolves deterministically with time $t$.

Now we switch on the resetting protocol, whereby the system goes back randomly in time to the initial high-temperature state with a nonzero rate $r$. This means that, between two successive resetting events, the system evolves by the standard Glauber dynamics mentioned above (1). If we now observe the system at a fixed time $t$, what matters is the time $\tau$ elapsed 
since the last resetting before $t$. This is because the system has evolved by the pure Glauber dynamics during the interval $[t-\tau, t]$. But since the resettings happen stochastically, the time $\tau$ itself is a random variable. As a result, any observable, such as the average magnetization, measured at time $t$ also becomes a random variable. One can express the distribution $P_{r}(m, t)$ of the average magnetization $m$ in the presence of resetting with rate $r$ by the simple renewal equation

$$
P_{r}(m, t)=r \int_{0}^{t} d \tau e^{-r \tau} P_{0}(m, \tau)+e^{-r t} P_{0}(m, t),
$$

where $P_{0}(m, \tau)=\delta(m-m(\tau))$ denotes the magnetization distribution in the absence of resetting $(r=0)$ since it evolves deterministically as $m(\tau)$. The second term in (2) corresponds to having no resetting up to time $t$ and the system evolves by the standard Glauber dynamics during $[0, t]$ and ends up with a magnetization $m$ at time $t$. The first term in (2) corresponds to the event that there is a resetting event at time $t-\tau$ which happens with probability $r d \tau$, followed by no resetting in the interval $[t-\tau, t]$ which occurs with probability $e^{-r \tau}$. During this interval of length $\tau$, the system evolves via the standard Glauber dynamics, and hence at time $t$, the magnetization is just $m(\tau)$. In the large time limit, the second term in (2) drops out and hence the stationary magnetization distribution is given by

$$
P_{r}^{\text {stat }}(m)=r \int_{0}^{\infty} d \tau e^{-r \tau} \delta(m-m(\tau)) .
$$

Given this simple renewal property (3), we need just to know the deterministic Glauber evolution $m(\tau)$ for all $\tau$ in the absence of resetting to characterize $P_{r}^{\text {stat }}(m)$.

\section{A. The case of the $1 d$ Ising model}

We start with the exactly solvable case on a $1 d$ lattice with $N$ sites and periodic boundary conditions in the absence of resetting. The one-point average $\left\langle s_{i}(t)\right\rangle$ evolves via [54]

$$
\frac{d}{d t}\left\langle s_{i}(t)\right\rangle=-\left\langle s_{i}(t)\right\rangle+\frac{\gamma}{2}\left[\left\langle s_{i-1}(t)\right\rangle+\left\langle s_{i+1}(t)\right\rangle\right],
$$

with initial condition $\left\langle s_{i}(0)\right\rangle=m_{0}$ for all $i$. Thus the average magnetization $m(t)=(1 / N) \sum_{i}\left\langle s_{i}(t)\right\rangle$ evolves via $d m(t) / d t=-(1-\gamma) m(t)$, whose solution is trivially

$$
m(t)=m_{0} e^{-(1-\gamma) t} ; \quad \gamma=\tanh (2 \beta J) .
$$

Substituting this solution (5) in Eq. (3), one obtains exactly

$$
P_{r}^{\text {stat }}(m)=\frac{r}{m_{0}(1-\gamma)}\left(\frac{m}{m_{0}}\right)^{\frac{r}{r^{*}(T)}-1}, \quad m \in\left[0, m_{0}\right],
$$

where

$$
r^{*}(T)=1-\gamma=1-\tanh \left(\frac{2 J}{k_{B} T}\right) .
$$

In this case $T_{c}=0$ and we have only the part of the phase diagram in Fig. 1 with $T \geqslant T_{c}$. The result in Eq. (6) clearly shows that, near $m=0, P_{r}^{\text {stat }}(m) \sim m^{\zeta}$ where the exponent $\zeta=r / r^{*}(T)-1$ varies continuously with temperature. Thus, $P_{r}^{\text {stat }}(m)$ either diverges [for $r<r^{*}(T)$ ] or vanishes [for $r>$ $\left.r^{*}(T)\right]$ as $m \rightarrow 0$. In the former case, $m_{\text {typ }}=0$ - this is the para phase. In contrast, for $r>r^{*}(T), m_{\mathrm{typ}}>0$ : This is the pseudoferro phase induced by resetting. We have also done numerical simulations in $d=1$ to verify our analytical prediction in Eq. (6) and found excellent agreement (see Fig. 3 in Appendix A).

\section{B. The case of the $2 d$ Ising model}

In $2 d$, the Ising model at equilibrium has a finite $T_{c} \approx$ 2.269 with the choice $J=k_{B}=1$. Unlike in $d=1$, the Glauber dynamics is not exactly solvable in $d=2$. However, using the well-established phenomenological behavior of $m(t)$, in particular at late times, in the renewal equation (3), we can make some predictions for the stationary magnetization distribution $P_{r}^{\text {stat }}(m)$ in various parts of the phase diagram in the $(T, r)$ plane in Fig. 1. We then verify these predictions with numerical simulations and find a very good agreement. Below we consider the three cases $T>T_{c}, T<T_{c}$, and $T=$ $T_{c}$ separately:

(1) $T>T_{c}$. We start with the paramagnetic phase $T>T_{c}$. In this case, the average magnetization for the pure Glauber dynamics is expected to decay at late times as $m(t) \sim a_{1} e^{-\lambda_{1} t}$, where the amplitude $a_{1}$ and the leading decay rate $\lambda_{1}$ both depend on temperature [58] and can be estimated very precisely from Monte Carlo simulations. This pure exponential decay of $m(t)$ holds only when $t \gg 1 / \Delta \lambda$, where $\Delta \lambda$ is the first gap in the relaxation spectrum. Substituting this functional form in Eq. (3) we get, for $r \ll \Delta \lambda$,

$$
P_{r}^{\text {stat }}(m) \approx \frac{r}{\lambda_{1} a_{1}^{\frac{r}{\lambda_{1}}}} m^{\frac{r}{\lambda_{1}}-1}, \quad m \in\left(0, a_{1}\right] .
$$

This is a good approximation at high temperature $T \gg T_{c}$, where $\Delta \lambda$ is large. In this case, $a_{1} \approx m_{0}$ and thus we recover qualitatively a similar distribution for $P_{r}^{\text {stat }}(m)(6)$ as in the $d=1$ case. In the case of $2 d$, for $T \gg T_{c}$, we then have $r^{*}(T)=\lambda_{1}$. Thus, as in the $d=1$ case, we have a crossover from the usual para phase for $r<r^{*}(T)$ to the pseudoferro phase for $r>r^{*}(T)$ across the crossover line $r^{*}(T)$ in the $(T, r)$ plane, for $T \gg T_{c}$. Our numerical simulations are completely consistent with this scenario. In Fig. 2(a), we plot the cumulative stationary distribution $F_{r}^{\text {stat }}(m)=\int_{0}^{m} P_{r}^{\text {stat }}\left(m^{\prime}\right) d m^{\prime}$ versus $m$ for three different resetting rates: one in the para phase (the top curve), one in the pseudoferro phase (bottom curve), and finally at the crossover line $r=r^{*}(T)$ (middle curve). In the last case, the probability distribution function (PDF) of the magnetization $P_{r^{*}}^{\text {stat }}(m)$ is uniform [from Eq. (8)] and hence the cumulative distribution function $(\mathrm{CDF}) F_{r^{*}}^{\mathrm{stat}}(m)$ increases linearly with $m$.

(2) $T<T_{c}$. In the ferro phase and in the absence of resetting, the magnetization density of the $2 d$ Ising model reaches a nonzero equilibrium value $m_{\mathrm{eq}}$ with a stretched exponential decay at late times [58],

$$
m(t) \approx m_{\mathrm{eq}} \pm a e^{-b t^{c}},
$$

where the parameters $a, b$, and $0<c<1$, not known analytically, need to be determined from simulations. In (9), the + and - signs are used in the case $m_{0}>m_{\mathrm{eq}}$ and $m_{0}<m_{\mathrm{eq}}$ respectively. When a constant resetting rate $r$ is introduced in the system, the stationary PDF $P_{r}^{\text {stat }}(m)$ is obtained from the general formula in Eq. (3), where for $m(\tau)$ we now use Eq. (9). The resulting $P_{r}^{\text {stat }}(m)$ is nontrivial and its detailed form is 


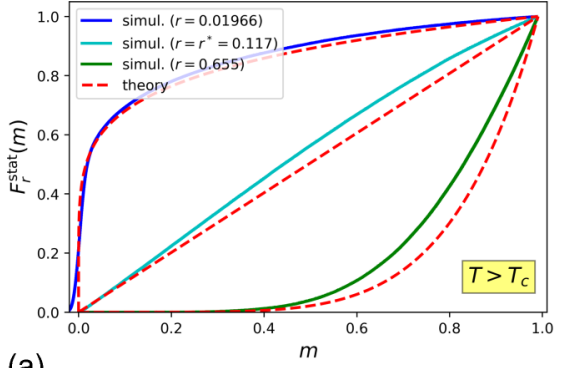

(a)

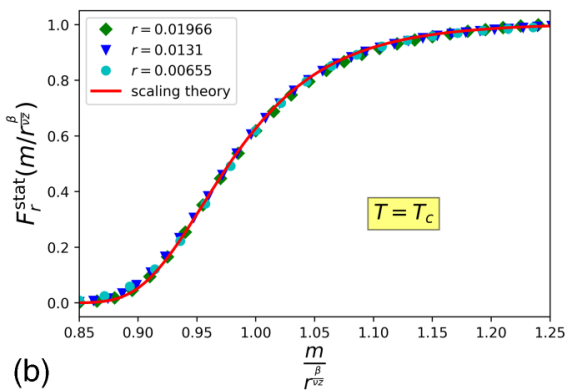

(b)

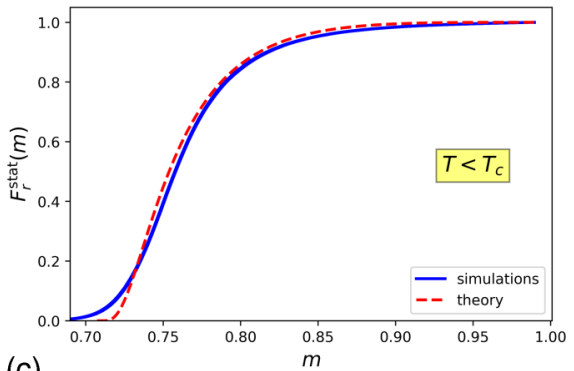

(c)

FIG. 2. Plot of the CDF $F_{r}^{\text {stat }}(m)=\int_{0}^{m} P_{r}^{\text {stat }}\left(m^{\prime}\right) d m^{\prime}$ vs $m$ in two dimensions, for different values of the resetting rate $r$, in (a) the para phase $T>T_{c}$, (b) at the critical point $T=T_{c} \approx 2.269$ (for $J=k_{B}=1$ ), and (c) in the ferro phase $T<T_{c}$. (a) The solid curves show the simulation results for $T=3.5, m_{0}=0.9905$, and for three different values of $r$, respectively for $r<r^{*}$ (top blue curve), $r=r^{*} \approx 0.117$ (middle light blue curve), and $r>r^{*}$ (bottom green curve). The red dashed lines correspond to our theoretical prediction obtained by integrating Eq. (8). The agreement with the theoretical predictions gets better for smaller $r$ as explained in the text. (b) Scaling collapse at $T=T_{c}$ of the CDF $F_{r}^{\text {stat }}(m) \approx H\left(m r^{-\beta /(v z)}\right)$, compared with the theoretical function $H(y)=e^{-\left(b_{c} / y\right)^{\nu z / \beta}}$ (red solid line) for three different small values of $r$. Here we used $\beta=1 / 8, v=1$ and $z \approx 2.17$ together with the estimated parameter $b_{c}=0.9576$. (c) CDF for $T=2.24<T_{c}$ with $m_{\mathrm{eq}}=0.70732$ and $r=0.00459$ (blue solid line), compared with the theoretical prediction [see the text and Eq. (B3) in Appendix B] shown by the dashed red line. All the simulations were performed on a $256 \times 256$ square lattice. One observes finite-size effects at the edges of the support.

discussed in Appendix B. For instance, a plot of the associated CDF is given in Fig. 2(c) for the case $m_{0}>m_{\mathrm{eq}}$ and compared to simulations, showing an excellent agreement. In this case, the PDF is supported over the finite interval $\left[m_{\mathrm{eq}}, m_{\mathrm{eq}}+a\right]$. It has nontrivial asymptotic behaviors at the edges. For example, near the lower edge, where $m \rightarrow m_{\text {eq }}^{+}, P_{r}^{\text {stat }}(m)$ vanishes faster than a power law as $P_{r}^{\text {stat }}(m) \sim \exp \{-B[-\ln ((m-$ $\left.\left.\left.\left.m_{\mathrm{eq}}\right) / a\right)\right]^{1 / c}\right\}$, where $0<c<1$ and $B=r b^{-1 / c}$.

(3) $T=T_{c}$. Exactly at the critical point, the magnetization $m(t)$, without resetting, has a nonmonotonic decay with time [59-61], which is not known analytically, except at short and long times. Hence, it is difficult to evaluate $P_{r}^{\text {stat }}(m)$ exactly from Eq. (3) for all $m$. However, when $r$ and $m$ are both small, one can use in Eq. (3) the late time form of $m(t) \approx b_{c} t^{-\phi}$, where the exponent $\phi=\beta /(v z)$ is related to the standard critical exponents defined earlier. We then find that there is a scaling regime as $m \rightarrow 0, r \rightarrow 0$ but with $m r^{-\phi}$ fixed where the distribution $P_{r}^{\text {stat }}(m)$ takes the scaling form

$$
P_{r}^{\text {stat }}(m) \approx r^{-\phi} G\left(m r^{-\phi}\right),
$$

where the scaling function $G(y)=A y^{-1-1 / \phi} e^{-\left(b_{c} / y\right)^{1 / \phi}}$, with $A=b_{c}^{1 / \phi} / \phi$, vanishes extremely rapidly as $y \rightarrow 0$. Consequently, the $\operatorname{CDF} F_{r}^{\text {stat }}(m)=\int_{0}^{m} P_{r}^{\text {stat }}\left(m^{\prime}\right) d m^{\prime} \approx H\left(m r^{-\phi}\right)$, where $H(y)=e^{-\left(b_{c} / y\right)^{1 / \phi}}$. This scaling behavior is verified numerically in Fig. 2(b), where $F_{r}^{\text {stat }}(m)$ shows a beautiful scaling collapse for three different values of $r$. The full distribution $P_{r}^{\text {stat }}(m)$ has still a finite support $m \in[0, \Gamma]$ where the upper cutoff $\Gamma$ depends on system parameters. While there is no strict gap at $m=0$ (as in the ferro phase), $P_{r}^{\text {stat }}(m)$ vanishes extremely rapidly as $m \rightarrow 0$. Thus, even in this resetting-induced NESS, there is a remnant signature of the equilibrium critical point $T_{c}$ that is manifest in this essential singularity near $m=0$.

\section{CONCLUSION}

To summarize, we have addressed a general question: How does resetting affect a magnetic system such as the short-range Ising model that, in equilibrium, exhibits a thermodynamic phase transition at $T=T_{c}(d>1)$ ? We have shown that the resetting can be realized in such systems using a rapid quench protocol of temperature and magnetic field. Resetting drives the system to a NESS with a nontrivial magnetization distribution and exhibits a rich phase diagram in the $(T, r)$ plane (see Fig. 1), including in particular the emergence of a pseudoferro phase for $T>T_{c}$ and $r>r^{*}(T)$ which has

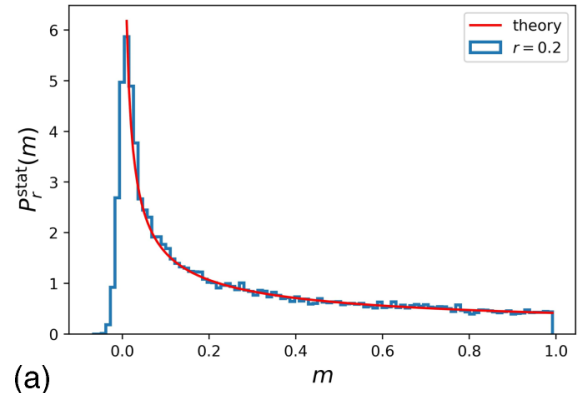

(a)

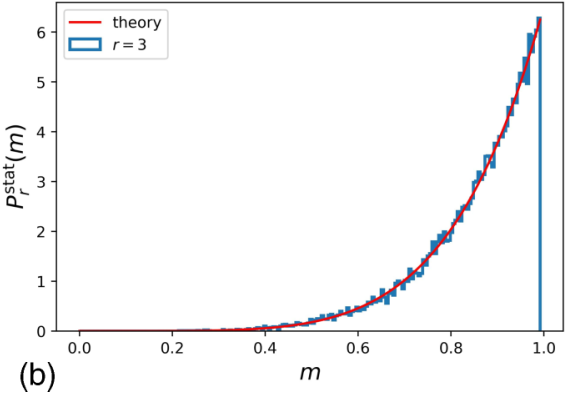

(b)

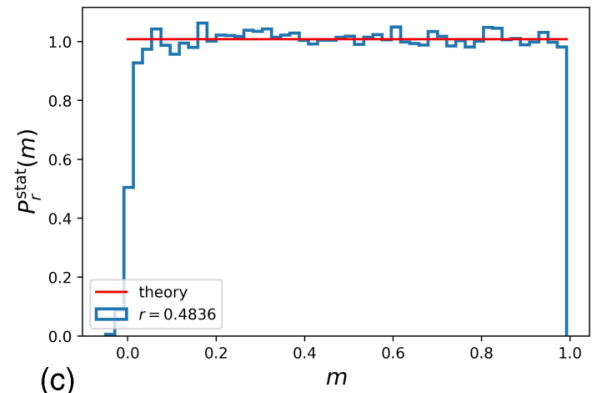

(c)

FIG. 3. Plot of the PDF $P_{r}^{\text {stat }}(m)$ for three different values of $r$ : (a) para phase with $r=0.2<r^{*}(T=3.5)=0.4836 \ldots$, (b) pseudoferro phase with $r=3>r^{*}(T=3.5)$, and (c) exactly on the crossover line $r=r^{*}(T=3.5)$. The red solid lines correspond to the theoretical result in Eq. (6), while the blue curves correspond to numerical simulations. The almost blue vertical lines at the edges of the distribution are due to finite-size effects. 
been characterized. The qualitative features of the phase diagram, established here for the $d=1$ and $d=2$ Ising model with Glauber dynamics, are also expected to hold in higher dimensions and for generic magnetic systems. It would be interesting to study the effect of a nonzero relaxation time $\tau_{R}$ on the stationary magnetization distribution. Finally, going beyond the magnetization distribution, it would be interesting to investigate the structure of the distribution of the two-point correlation functions in this resetting-induced NESS.

\section{APPENDIX A: RESETTING IN THE 1d ISING MODEL: MONTE CARLO SIMULATIONS}

For the $1 d$ Ising model, $T_{c}=0$, and hence there is no ferromagnetic phase for $T>0$. Hence, in the phase diagram of Fig. 1 , we have only the phase $T \geqslant T_{c}=0$. The stationary probability distribution of the average magnetization in the $1 d$ Ising model in the presence of resetting is given by Eq. (6) and reads

$$
P_{r}^{\text {stat }}(m)=\frac{r}{m_{0}(1-\gamma)}\left(\frac{m}{m_{0}}\right)^{\frac{r}{r^{r}(T)}-1}, \quad m \in\left[0, m_{0}\right],
$$

where

$$
r^{*}(T)=1-\gamma=1-\tanh \left(\frac{2 J}{k_{B} T}\right) .
$$

We have performed Monte Carlo simulations on a onedimensional lattice of length $N=10000$ at temperature $T=$ 3.5 (setting $J=k_{B}=1$ ) to verify our analytical results. Simulation data are collected by averaging over 3000 independent realizations. The fixed initial configuration to which the system is reset at a constant rate $r$ has magnetization $m_{0}=0.992$. Figure 3 presents the plot of the PDF $P_{r}^{\text {stat }}(m)$ for three different values of $r$, showing the excellent agreement between theory (red line) and Monte Carlo simulations (plotted in blue).

We perform the Glauber dynamics of the $1 d$ Ising model at temperature $T=3.5$, for which $r^{*}(T=3.5)=1-$ $\tanh (2 / 3.5)=0.4836$. Hence, for $r<r^{*}(T=3.5), P_{r}^{\text {stat }}(m)$ diverges as $m \rightarrow 0$. This is clearly shown in Fig. 1(a), where, in the presence of a resetting rate $r=0.2$, the typical value of the average magnetization is $m_{\mathrm{typ}}=0$ : This regime is the para phase. In contrast, for $r>r^{*}(T=3.5)$, a pseudoferro phase emerges, where $m_{\mathrm{typ}}>0$ and $P_{r}^{\text {stat }}(0)=0$, as shown in Fig. 1(b). Exactly on the crossover line $r=r^{*}(T)$, Eq. (6) predicts a uniform distribution for $P_{r}^{\text {stat }}(m)$ in $\left[0, m_{0}\right]$ : This is numerically verified in Fig. 1(c).

It is also interesting to know how the first moment of $P_{r}^{\text {stat }}(m)$ depends on the resetting rate $r$. This can be computed easily as

$$
\overline{m(r)}=r \int_{0}^{\infty} d \tau e^{-r \tau} m_{0} e^{-(1-\gamma) t}=\frac{r}{r+r^{*}(T)} m_{0},
$$

where $r^{*}(T)=1-\gamma$. We have measured this quantity $\overline{m(r)}$ for different values of $r$ from our numerical simulations. They are plotted in blue in Fig. 4, showing an excellent agreement with Eq. (A3). However, as discussed in the text, the average magnetization is not enough to characterize the stationary state under resetting and one needs the full distribution $P_{r}^{\text {stat }}(m)$.

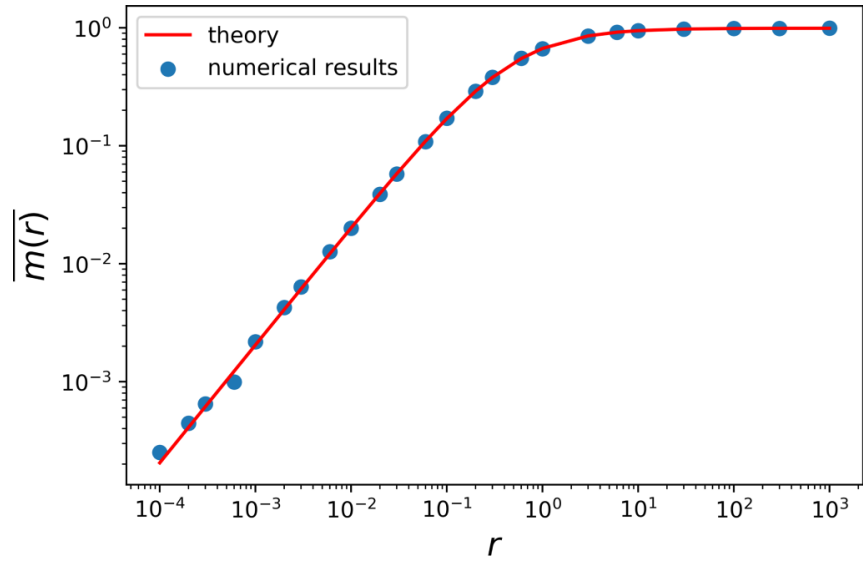

FIG. 4. Plot of the first moment $\overline{m(r)}$ as a function of $r$. Numerical data and Eq. (A3) are plotted in blue and red respectively.

\section{APPENDIX B: SCALING PREDICTIONS FOR $P_{r}^{\text {stat }}(m)$ IN THE THREE TEMPERATURE REGIMES FOR THE ISING MODEL WITH RESETTING IN $\boldsymbol{d} \geqslant 2$}

For the Ising model in $d \geqslant 2$, the critical temperature $T_{c}$ is finite. Hence, in the phase diagram of Fig. 1, we will have all the three phases. Unlike in $1 d$, the Glauber dynamics is not exactly solvable in $d \geqslant 2$, even in the absence of resetting. In this section, we provide some general scaling arguments to predict scaling forms for $P_{r}^{\text {stat }}(m)$ for $T>T_{c}, T<T_{c}$, and $T=T_{c}$, using the renewal equation (3) which reads

$$
P_{r}^{\text {stat }}(m)=r \int_{0}^{\infty} d \tau e^{-r \tau} \delta(m-m(\tau))
$$

\section{The high-temperature phase $T>T_{c}$}

In the high-temperature phase, the average magnetization, quite generally, decays with time as $m(t)=\sum_{i} a_{i} e^{-\lambda_{i} t}$, where $\lambda_{1}<\lambda_{2}<\cdots$ denotes the eigenvalues associated to the relaxation spectrum. At late times, this is dominated by the lowest eigenvalue $\lambda_{1}$, i.e., $m(t) \approx a_{1} e^{-\lambda_{1} t}$. This pure exponential decay is thus valid when $t \gg 1 / \Delta \lambda$, where $\Delta \lambda=\lambda_{2}-\lambda_{1}$ is the first gap in the relaxation spectrum. Substituting this pure exponential form in Eq. (B1) we get, for $r \ll \Delta \lambda$,

$$
\begin{aligned}
P_{r}^{\text {stat }}(m) & \approx r \int_{0}^{\infty} d \tau e^{-r \tau} \delta\left(m-a_{1} e^{-\lambda_{1} \tau}\right) \\
& \approx \frac{r}{\lambda_{1} a_{1}^{\frac{r}{\lambda_{1}}}} m^{\frac{r}{\lambda_{1}}-1}, \quad m \in\left(0, a_{1}\right] .
\end{aligned}
$$

This is Eq. (8) and it is valid for $T \gg T_{c}$, such that the first gap $\Delta \lambda$ is large.

\section{The low-temperature phase $T<T_{c}$}

In the low-temperature phase, the average magnetization $m$ approaches the equilibrium magnetization $m_{\mathrm{eq}}>0$ as a stretched exponential at late times [58], i.e., $m(t) \approx m_{\mathrm{eq}} \pm$ $a e^{-b t^{c}}$, where the + and - signs are used in the cases $m_{0}>$ $m_{\mathrm{eq}}$ and $m_{0}<m_{\mathrm{eq}}$ respectively. 

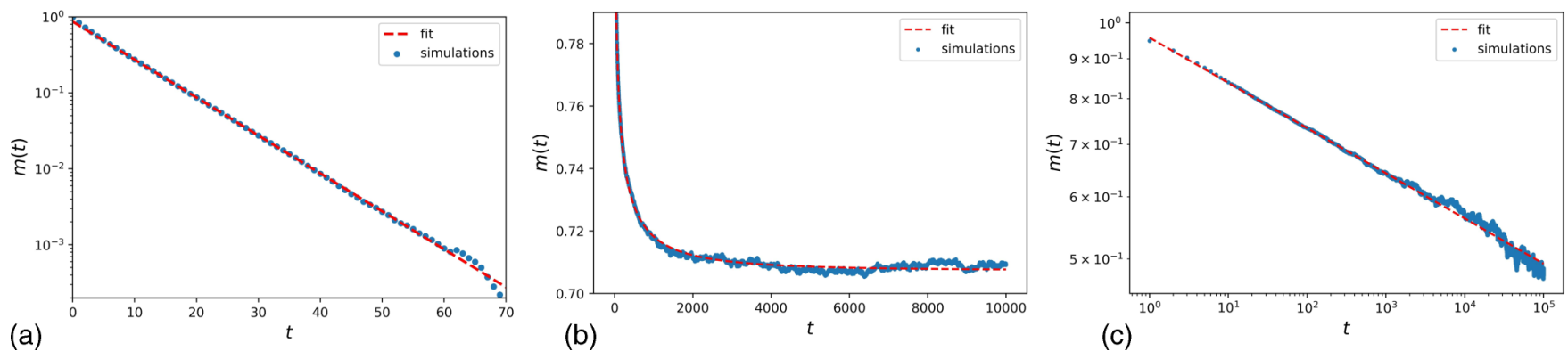

FIG. 5. Fit of $m(t)$ in the three temperature regimes. For $T<T_{c}$ and $T=T_{c}$, it is necessary to consider many more time steps to obtain an accurate fit, since the decay to the equilibrium magnetization is slower than the one for $T>T_{c}$.

Inserting this expression for $m(t)$ in Eq. (B1), we obtain for $r \ll \Delta \lambda$

$$
\begin{aligned}
P_{r}^{\text {stat }}(m) \approx & r \int_{0}^{\infty} d \tau e^{-r \tau} \delta\left(m-m_{\mathrm{eq}} \mp a e^{-b \tau^{c}}\right) \\
\approx & \frac{r}{b c} \int_{0}^{a} d x \frac{1}{\left[\frac{1}{b} \ln \left(\frac{a}{x}\right)\right]^{\frac{c-1}{c}} x} \\
& \times \exp \left\{-r\left[\frac{1}{b} \ln \left(\frac{a}{x}\right)\right]^{\frac{1}{c}}\right\} \delta\left(m-m_{\mathrm{eq}} \mp x\right) \\
\approx & \frac{r}{b c[f(m)]^{\frac{c-1}{c}}\left|m-m_{\mathrm{eq}}\right|} \exp \left\{-r[f(m)]^{\frac{1}{c}}\right\},
\end{aligned}
$$

where $f(m)=\frac{1}{b} \ln \left(\left|\frac{a}{m-m_{\mathrm{eq}}}\right|\right)$. Its support is $\left(m_{\mathrm{eq}}, m_{\mathrm{eq}}+a\right]$ if $m_{0}>m_{\mathrm{eq}}$ or $\left[m_{\mathrm{eq}}-a, m_{\mathrm{eq}}\right)$ if $m_{0}<m_{\mathrm{eq}}$. In the second equality, we have used $x=a e^{-b \tau^{c}}$.

\section{At the critical point $T=T_{c}$}

At $T=T_{c}$, the late time evolution of $m(t)$ in the absence of resetting follows a power law decay to 0 since the spectrum is gapless. In this case, at late times, $m(t) \approx b_{c} t^{-\phi}$, where the exponent is $\phi=\beta /(v z)$ [61]. More precisely, this power law decay holds for $t<L^{z}$, where $L$ is the linear size of the system. This condition is fulfilled in our simulations since $\frac{1}{r_{\min }} \ll L^{z}$, where $r_{\min }$ is the smallest resetting rate used in our simulations. Using Eq. (B1), we obtain for $r \ll 1$

$$
\begin{aligned}
P_{r}^{\mathrm{stat}}(m) & \approx r \int_{0}^{\infty} d \tau e^{-r \tau} \delta\left(m-b_{c} t^{-\phi}\right) \\
& \approx r \frac{b_{c}^{\frac{1}{\phi}}}{\phi} \int_{0}^{\infty} d x \frac{1}{x^{\frac{\phi+1}{\phi}}} \exp \left[-r\left(\frac{b_{c}}{x}\right)^{\frac{1}{\phi}}\right] \delta(m-x) \\
& \approx \frac{r b_{c}^{\frac{1}{\phi}}}{\phi m^{\frac{\phi+1}{\phi}}} \exp \left[-r\left(\frac{b_{c}}{m}\right)^{\frac{1}{\phi}}\right]
\end{aligned}
$$

where in the second equality we made the change of variable $x=b_{c} t^{-\phi}$. In Eq. (B4), if we set the scaling variable $y=$ $m r^{-\phi}$, then $P_{r}^{\text {stat }}(m)$ takes the scaling form given in Eq. (10).

\section{APPENDIX C: NUMERICAL ESTIMATE OF THE PARAMETERS IN THE TIME EVOLUTION $m(t)$ OF THE $2 d$ ISING MODEL}

In order to use Eq. (3) and compute $P_{r}^{\text {stat }}(m)$, we need to estimate the parameters in the time evolution of $m(t)$. To this end, we have done Monte Carlo simulations of the Glauber dynamics in the three temperature regimes on a $256 \times 256$ square lattice with the choice $J=k_{B}=1$ and with $m_{0}=$ 0.9905 as the magnetization of the starting configuration at time $t=0$. Figure 5 shows the fit of $m(t)$ in the paramagnetic phase [Fig. 3(a)], in the ferromagnetic phase [Fig. 3(b)], and at the critical temperature [Fig. 3(c)]. The blue dots (simulation data) are obtained by averaging over 4770, 490, and 70 independent realisations at temperatures $T=3.5, T=$ 2.24, and $T=T_{c}=2.269$ respectively. The red dashed lines are obtained by fitting the well-established phenomenological behavior of $m(t)$ in the three temperature regimes to the numerical data.

As mentioned above, for $T>T_{c}$, the average magnetization decays at late times as $m(t) \approx a_{1} e^{-\lambda_{1} t}$. The fit shown in Fig. 3(a) provides the values $a_{1}=0.889$ and $\lambda_{1}=0.117$ for $T=3.5$. For the low-temperature phase, we set $T=2.24<$ $T_{c}=2.269$ and in Fig. 3(b) we show the fit of the functional form $m(t) \approx m_{\mathrm{eq}}+a e^{-b t^{c}}$ with estimated parameter values $a=0.283, b=0.37$, and $c=0.316$. At the critical temperature $T_{c}=2.269$, the fit, $m(t)=b_{c} t^{-\phi}$, shown in Fig. 3(c) provides an estimation of the parameters $b_{c}=0.9576$ and $\phi=0.0576$. Using the relation $\phi=\beta /(v z)$, and the known exact values of $\beta=\frac{1}{8}$ and $v=1$, one gets $z=1 /(8 \phi) \approx$ 2.17 , which is in excellent agreement with previous largescale simulations [58].

\section{APPENDIX D: FIRST MOMENT OF $P_{r}^{\text {stat }}$ IN THE $2 d$ ISING MODEL}

A quantity of interest that clearly depends on the resetting rate $r$ is the first moment of $P_{r}^{\text {stat }}(m)$ in the $2 d$ Ising model. Similarly to the approach followed for the $1 d$ case, we can easily compute it for $T>T_{c}, T<T_{c}$, and $T=T_{c}$ as

$$
\overline{m(r)}=r \int_{0}^{\infty} d \tau e^{-r \tau} m(\tau),
$$

where $m(t)$ is the time evolution of the average magnetization in the Glauber dynamics which has been estimated in the previous section. 

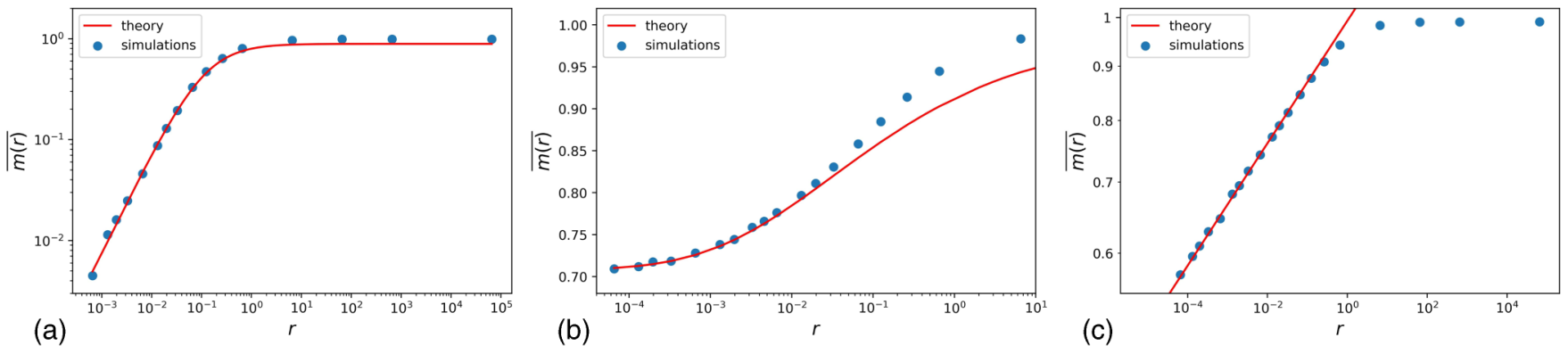

FIG. 6. Plot of $\overline{m(r)}$ for $T>T_{c}, T<T_{c}$, and $T=T_{c}$. The red lines are the theoretical results [see Eqs. (D2)-(D4)], while the blue dots come from Monte Carlo simulations.

For $T>T_{c}$, using $m(t) \approx a_{1} e^{-\lambda_{1} t}$ at late times, the average magnetization in the stationary state is given, for small $r$ [so that we can use the pure exponential late time decay of $m(t)]$, by

$$
\overline{m(r)} \approx r \int_{0}^{\infty} d \tau e^{-r \tau} a_{1} e^{-\lambda_{1} \tau}=\frac{r}{r+\lambda_{1}} a_{1},
$$

similarly to the one-dimensional case in Eq. (A3).

For $T<T_{c}$, using $m(t) \approx m_{\mathrm{eq}} \pm a e^{-b t^{c}}, \overline{m(r)}$ takes the form, for small $r$,

$$
\begin{aligned}
\overline{m(r)} & =r \int_{0}^{\infty} d \tau e^{-r \tau}\left(m_{\mathrm{eq}}+a e^{-b \tau^{c}}\right) \\
& =m_{\mathrm{eq}}+r a \int_{0}^{\infty} d \tau e^{-r \tau-b \tau^{c}},
\end{aligned}
$$

where the last integral can be estimated numerically very accurately.

At the critical temperature $T_{c}$, using $m(t) \approx b_{c} t^{-\phi}$, the average magnetization in the stationary state reads, for small $r$,

$$
\overline{m(r)}=r \int_{0}^{\infty} d \tau e^{-r \tau} b_{c} \tau^{-\phi}=b_{c} \Gamma(1-\phi) r^{\phi},
$$

where $\Gamma(z)$ is the $\Gamma$ function.

We have performed Monte Carlo simulations with different values of $r$ to verify the dependence of $\overline{m(r)}$ on $r$. The agreement is excellent in all the three phases [Figs. 6(a)-6(c)], especially for small values of $r$. This is consistent with the fact that we have taken the late time evolution of $m(t)$ in Eq. (D1), which holds only for small $r$.

\section{APPENDIX E: GENERAL EXPRESSION OF THE CUMULATIVE DISTRIBUTION}

In the main text, using renewal theory, we have obtained the expression of the nonequilibrium stationary PDF of the average magnetization in the presence of a resetting rate $r$ :

$$
P_{r}^{\text {stat }}(m)=r \int_{0}^{\infty} d \tau e^{-r \tau} \delta(m-m(\tau)),
$$

where $m(\tau)$ denotes the deterministic Glauber evolution of the average magnetization. It is easy to compute exactly the CDF

$$
F_{r}^{\text {stat }}(m)=\int_{0}^{m} P_{r}^{\text {stat }}\left(m^{\prime}\right) d m^{\prime}
$$

that we plot in Fig. 2. We can write $P_{r}^{\text {stat }}(m)$ as a slightly more general expression

$$
P_{r}^{\text {stat }}(m)=\int_{0}^{\infty} d \tau f(\tau) \delta(m-g(\tau)),
$$

where, of course, in our case $f(\tau)=r e^{-r \tau}$ and $g(\tau)=m(\tau)$. Then, making the change of variable $z=g(\tau)$, we can write the previous integral as

$$
\begin{aligned}
P_{r}^{\text {stat }}(m) & =\int_{g(0)}^{g(\infty)} \frac{1}{g^{\prime}\left(g^{-1}(z)\right)} f\left(g^{-1}(z)\right) \delta(m-z) d z \\
& =\frac{1}{\left|g^{\prime}\left(g^{-1}(m)\right)\right|} f\left(g^{-1}(m)\right) .
\end{aligned}
$$

Since $f^{\prime}(t)=-r f(t)$, the corresponding CDF is given by

$$
F_{r}^{\text {stat }}(m)= \pm \frac{1}{r} f\left(g^{-1}(m)\right)+\text { const }
$$

where the sign is + and const $=0$ if $g$ is a decreasing function of time, while the sign is - and const $=1$ if $g$ is an increasing function of time. Since in our case $f(t)=r e^{-r t}$, Eq. (E5) reduces to

$$
F_{r}^{\text {stat }}(m)= \pm e^{-r\left(g^{-1}(m)\right)}+\text { const. }
$$

This is the CDF that we plot in the main text, with the use of different functions $g$ in the three temperature regimes. The derivation of Eq. (E6) is based on two properties of the functions $f$ and $g$. The first one is that $f$ and its time derivative $f^{\prime}$ are proportional, since $f$ is an exponential function. The second property is that the function $g$, which describes the (deterministic) time evolution of the average magnetization, is a monotonic function and, therefore, is invertible. In particular, this property of the function $g$ is valid in the time window we considered for all the three different temperature regimes (paramagnetic and ferromagnetic phases and at the critical temperature).

\section{APPENDIX F: NUMERICAL ESTIMATE OF $r^{*}(T)$ IN THE PARAMAGNETIC PHASE OF THE $2 d$ ISING MODEL}

The exact expression of the crossover line $r^{*}(T)$ in the $1 d$ Ising model is given by Eq. (7) as $r^{*}(T)=1-\tanh \left(\frac{2 J}{k_{B} T}\right)$. It is then interesting to know if a similar curve can be obtained, at least numerically, also in the two-dimensional 


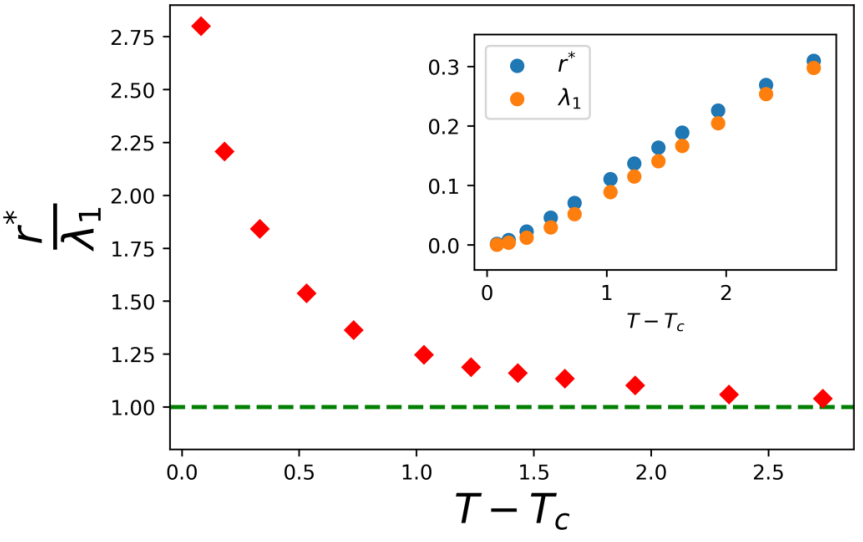

FIG. 7. The ratio $\frac{r^{*}}{\lambda_{1}}$ and, in the inset, the values of $\lambda_{1}$ and $r^{*}$ are estimated with Monte Carlo simulations at different temperatures above $T_{c}$.

case. For several temperatures $T>T_{c}$, we perform numerical simulations of the $2 d$ Glauber dynamics in the presence of several resetting rates $r$ and detect the value $r^{*}(T)$ that makes the resulting PDF $P_{r^{*}}^{\text {stat }}(m)$ more similar to the uniform distribution in $\left[0, m_{0}\right]$. We thus expect to obtain a curve $r^{*}(T)$ that divides the para phase from the pseudoferro phase as depicted in Fig. 1.

If the time evolution of $m(t)$ was given by a simple exponential decay to 0 , then we would find that $r^{*}(T)=$ $\lambda_{1}(T)$, as in the one-dimensional case. However, this is not the case, since this time dependence holds only at late times. Indeed, the time evolution of the average magnetization in the paramagnetic phase is given by

$$
m(t)=\sum_{i=1}^{N} a_{i} e^{-\lambda_{i} t},
$$

where $\lambda_{1}<\lambda_{2}<\cdots<\lambda_{N}$ constitute the whole relaxation spectrum, $a_{i}$ are coefficients, and $N=2^{L^{2}}$ is the total number of spin configurations in a $L \times L$ lattice. Equation (F1) is valid for any time $t$ and is therefore a generalization of the simple exponential decay. In the long-time limit, the two time evolutions coincide, because only the minimum value among $\lambda_{i}$ 's (i.e., $\lambda_{1}$ ) survives as $t \rightarrow \infty$.

In principle, $N$ should be equal to the total number of spin configurations. Of course, to make a reasonable fit, this number is too big: Therefore, we choose to take $N=3$; that is, we approximate $m(t)$ to be the sum of the three exponentials with the smallest decay rates. As a consequence, from the fit of $m(t)$ we estimate six parameters, which are $a_{i}$ and $\lambda_{i}$ for $i=1,2,3$.

It is therefore interesting to compare the curve $r^{*}(T)$ obtained as illustrated above with the value $\lambda_{1}(T)$ obtained by fitting this $m(t)$ to numerical data, for different temperatures $T>T_{c}$. The inset of Fig. 7 shows the values of $r^{*}$ and $\lambda_{1}$ for different temperatures above $T_{c}$. Note that $r^{*}(T)>\lambda_{1}(T)$, $\forall T>T_{c}$. In particular, if we imagine to draw a line connecting all the blue dots, we obtain the red dashed line in the phase diagram in Fig. 1. In the main plot of Fig. 7 the ratio $r^{*} / \lambda_{1}$ is plotted for different temperatures above $T_{c}$, using the same data points of the inset. We see that this ratio converges to 1 as the temperature $T$ increases, proving that, when $T \gg T_{c}$, the approximation $r^{*}=\lambda_{1}$ (which is the exact relation found in the $1 d$ case) is correct.
[1] For a recent review on resetting, see M. R. Evans, S. N. Majumdar, and G. Schehr, J. Phys. A 53, 193001 (2020).

[2] O. Bénichou, C. Loverdo, M. Moreau, and R. Voituriez, Rev. Mod. Phys. 83, 81 (2011).

[3] M. R. Evans and S. N. Majumdar, Phys. Rev. Lett. 106, 160601 (2011).

[4] M. R. Evans and S. N. Majumdar, J. Phys. A: Math. Theor. 44, 435001 (2011).

[5] M. R. Evans, S. N. Majumdar, and K. Mallick, J. Phys. A: Math. Theor. 46, 185001 (2013).

[6] J. Whitehouse, M. R. Evans, and S. N. Majumdar, Phys. Rev. E 87, 022118 (2013).

[7] M. Montero and J. Villarroel, Phys. Rev. E 87, 012116 (2013).

[8] M. R. Evans and S. N. Majumdar, J. Phys. A: Math. Theor. 47, 285001 (2014).

[9] S. Reuveni, M. Urbakh, and J. Klafter, Proc. Natl. Acad. Sci. USA 111, 4391 (2014).

[10] L. Kusmierz, S. N. Majumdar, S. Sabhapandit, and G. Schehr, Phys. Rev. Lett. 113, 220602 (2014).

[11] L. Kusmierz and E. Gudowska-Nowak, Phys. Rev. E 92, 052127 (2015).

[12] T. Rotbart, S. Reuveni, and M. Urbakh, Phys. Rev. E 92, 060101(R) (2015)
[13] C. Christou and A. Schadschneider, J. Phys. A 48, 285003 (2015).

[14] A. Pal, A. Kundu, and M. R. Evans, J. Phys. A 49, 225001 (2016).

[15] S. Reuveni, Phys. Rev. Lett. 116, 170601 (2016).

[16] A. Pal and S. Reuveni, Phys. Rev. Lett. 118, 030603 (2017).

[17] M. Montero and A. Masó-Puigdellosas, J. Villarroel Eur. Phys. J. B 90, 176 (2017).

[18] D. Boyer, M. R. Evans, and S. N. Majumdar, J. Stat. Mech. (2017) 023208.

[19] A. Chechkin and I. M. Sokolov, Phys. Rev. Lett. 121, 050601 (2018).

[20] S. Belan, Phys. Rev. Lett. 120, 080601 (2018).

[21] A. Pal and V. V. Prasad, Phys. Rev. Research 1, 032001 (2019).

[22] A. Pal, Phys. Rev. E 91, 012113 (2015).

[23] C. Maes and T. Thiery, J. Phys. A 50, 415001 (2017).

[24] A. Falcón-Cortés, D. Boyer, L. Giuggioli, and S. N. Majumdar, Phys. Rev. Lett. 119, 140603 (2017).

[25] S. N. Majumdar, S. Sabhapandit, and G. Schehr, Phys. Rev. E 91, 052131 (2015)

[26] J. Fuchs, S. Goldt, and U. Seifert, Europhys. Lett. 113, 60009 (2016).

[27] A. Nagar and S. Gupta, Phys. Rev. E 93, 060102(R) (2016). 
[28] É. Roldán, A. Lisica, D. Sánchez-Taltavull, and S. W. Grill, Phys. Rev. E 93, 062411 (2016).

[29] A. Lisica, C. Engel, M. Jahnel, É. Roldán, E. A. Galburt, P. Cramer, and S. W. Grill, Proc. Natl. Acad. Sci. USA 113, 2946 (2016).

[30] A. O. Gautestad and I. Mysterud, Am. Nat. 165, 44 (2005).

[31] A. O. Gautestad and I. Mysterud, Ecol. Complex. 3, 44 (2006).

[32] D. Boyer and C. Solis-Salas, Phys. Rev. Lett. 112, 240601 (2014).

[33] D. Boyer and J. C. R. Romo-Cruz, Phys. Rev. E 90, 042136 (2014).

[34] S. N. Majumdar, S. Sabhapandit, and G. Schehr, Phys. Rev. E 92, 052126 (2015).

[35] D. Boyer and I. Pineda, Phys. Rev. E 93, 022103 (2016).

[36] M. Villen-Altramirano and J. Villen-Altramirano, in Queueing Performance and Control in ATM, edited by J. W. Cohen and C. D. Pack (North-Holland, Amsterdam, London, 1991).

[37] M. Luby, A. Sinclair, and D. Zuckerman, Inf. Proc. Lett. 47, 173 (1993).

[38] A. Montanari and R. Zecchina, Phys. Rev. Lett. 88, 178701 (2002).

[39] H. Tong, C. Faloutsos, and J.-Y. Pan, Knowl. Inf. Syst. 14, 327 (2008).

[40] K. Avrachenkov, A. Piunovskiy, and Y. Zhang, J. Appl. Prob. 50, 960 (2013).

[41] J. H. Lorenz, SOFSEM 2018: Theory and Practice of Computer Science, edited by A. Tjoa et al., Lecture Notes in Computer Science Vol. 10706 (Springer, Cham, 2018).

[42] A. Scacchi and A. Sharma, Mol. Phys. 116, 460 (2017).

[43] M. R. Evans and S. N. Majumdar, J. Phys. A: Math. Theor. 51, 475003 (2018).
[44] J. Masoliver, Phys. Rev. E 99, 012121 (2019).

[45] S. Ghosh, B. Mishra, A. B. Kolomeisky, and D. Chowdhury, J. Stat. Mech. (2018) 123209.

[46] T. T. da Silva and M. D. Fragoso, J. Phys. A: Math. Theor. 51, 505002 (2018).

[47] B. Mukherjee, K. Sengupta, and S. N. Majumdar, Phys. Rev. B 98, 104309 (2018).

[48] D. C. Rose, H. Touchette, I. Lesanovsky, and J. P. Garrahan, Phys. Rev. E 98, 022129 (2018).

[49] X. Durang, M. Henkel, and H. Park, J. Phys. A: Math. Theor 47, 045002 (2014).

[50] S. Gupta, S. N. Majumdar, and G. Schehr, Phys. Rev. Lett. 112 220601 (2014).

[51] U. Basu, A. Kundu, and A. Pal, Phys. Rev. E 100, 032136 (2019).

[52] S. Karthika and A. Nagar, J. Phys. A: Math. Theor. 53, 115003 (2020)

[53] P. Grange, J. Phys. Commun. 4, 045006 (2020).

[54] R. J. Glauber, J. Math. Phys. 4, 294 (1963).

[55] M. R. Evans and S. N. Majumdar, J. Phys. A 52, 01LT01 (2019).

[56] A. Pal, Ł. Kuśmierz, and S. Reuveni, Phys. Rev. E 100, 040101(R) (2019); New J. Phys. 21, 113024 (2020).

[57] D. Gupta, C. A. Plata, and A. Pal, Phys. Rev. Lett. 124, 110608 (2020).

[58] D. Stauffer, Physica A (Amsterdam, Neth.) 244, 344 (1997).

[59] H. K. Janssen, B. Schaub, and B. Schmittmann, Z. Phys. B 73, 539 (1989).

[60] B. Zheng, Int. J. Mod. Phys. B 12, 1419 (1998).

[61] P. Calabrese and A. Gambassi, J. Phys. A: Math. Gen. 38, R133 (2005). 